\title{
Psychometric properties of the Persian version of the nutritional form for the elderly (NUFFE) in nursing home residents
}

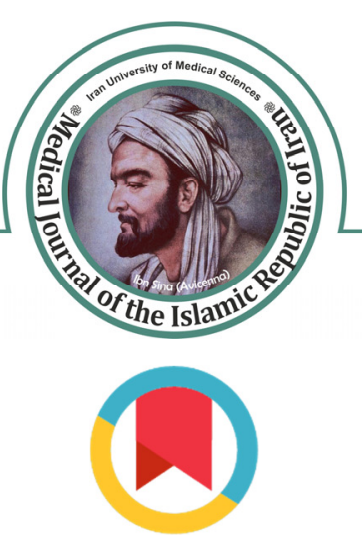

\begin{abstract}
Farshad Sharifi ${ }^{1}$, Mojde Mirarefin ${ }^{2}$, Mahtab Alizadeh-Khoei ${ }^{* 1,3}$, Neda Nazari ${ }^{1,4}$, Baharak Najafi ${ }^{1}$, Hossein Fakhrzadeh ${ }^{1}$, Seyed Masoud Arzaghi ${ }^{1}$, Ulrika Söderhamn ${ }^{5}$, Fahimeh Taati ${ }^{1}$, Neda Mehrdad ${ }^{6}$
\end{abstract}

Received: 17 Oct 2017

Published: 30 Oct 2018

\section{Abstract}

Background: The Nutritional Form for the Elderly (NUFFE) is a newly developed tool. This study aimed to carry out a psychometric evaluation of the Persian version of NUFFE (NUFFE-P) among nursing home residents.

Methods: Nursing home participant's aged $\geq 60$ years $(n=97)$ were enrolled. The inclusion criteria were residency for at least 6 months in the nursing home, and ability to communicate. Exclusion criteria included cognitive impairment, having depressed mood, severe hearing loss, problems in upper and lower extremities also, history of hospital admission during 6 months before enrollment. Anthropometric measures, laboratory tests, three-day food intake, NUFFE-P version, Mini Nutritional Assessment (MNA), Barthel Index (BI) and Geriatric Depression Scale (GDS-15) were assessed. The relationship between the NUFFE-P and MNA scores was considered as concurrent validity.

Results: The Cronbach's alpha coefficient of NUFFE-P tool was 0.76 . The intraclass correlation coefficient for the total score between two raters obtained 0.98 (CI 0.97-0.99). The correlation coefficient between the NUFFE-P and the MNA scores was -0.75 $(\mathrm{p}<0.01)$. Four factors were extracted for the NUFFE-P in an exploratory factor analyses. Sensitivity $69.8 \%$ and $100 \%$ and specificity $75.7 \%$ and $85.6 \%$ were achieved to detect elderly at medium risk (cutoff $=6$ ), and at high risk of under-nutrition (cutoff $=11$ ) respectively.

Conclusion: The NUFFE-P has sufficient psychometric properties in nutritional status screening among the Iranian elderly nursing homes residents.

Keywords: Elderly, Nutrition assessment, Sensitivity, Specificity

Conflicts of Interest: None declared

Funding: University of Social Welfare and Rehabilitation Sciences

\section{*This work has been published under CC BY-NC-SA 1.0 license.}

Copyright $\odot$ Iran University of Medical Sciences

Cite this article as: Sharifi F, Mirarefin M, Alizadeh-Khoei M, Nazari N, Najafi B, Fakhrzadeh H, Arzaghi SM, Söderhamn U, Taati F, Mehrdad N Psychometric properties of the Persian version of the nutritional form for the elderly (NUFFE) in nursing home residents. Med $J$ Islam Repub Iran. 2018 (30 Oct);32:105. https://doi.org/10.14196/mjiri.32.105

\section{Introduction}

Elderly people are at a higher risk of nutritional disturbance than younger adults because of the several alterations in mental and physical function (1). Furthermore, malnutrition is a common health problem in geriatric settings, i.e. hospitals, nursing homes, and home care (2). On the other hand, malnutrition may be associated with a high

Corresponding author: Dr Mahtab Alizadeh-Khoei, alizadeh-m@sina.tums.ac.ir

1. Elderly Health Research Center, Endocrinology and Metabolism Population Sciences Institute, Tehran University of Medical Sciences, Tehran, Iran

2. Division of Public and Behavioral Health, Nevada Department of Health and Human Services, Carson City, USA

3. Geriatric \& Gerontology Department, Medical school, Tehran University of Medical Sciences, Tehran, Iran

4. Kahrizak Charity Foundation Nursing Home, Tehran, Iran

5. Center of Caring Research - Southern Norway, University of Agder, Kristiansand, Norway

6. Endocrinology and Metabolism Research Center, Endocrinology and Metabolism Clinical Sciences Institute, Tehran University of Medical Sciences, Tehran, Iran incidence rate of morbidity and mortalities (1). Undernourishment may also have a role in the quality of life reduction among older people, even after adjusting for the severity of comorbidities (3). The prevalence of malnourishment among elderly in long-term care services was reported to be $10 \%$ and $85 \%$, respectively (4). The preva-

$\uparrow$ What is "already known" in this topic:

Nutritional assessment is an important domain of health evaluation in elderly. The Nutritional Form for the Elderly (NUFFE) is an instrument for nutritional screening that is easily applicable without special nutritional training.

\section{$\rightarrow$ What this article adds:}

The developed Persian version of NUFFE is a valid and reliable tool in nutritional screening among Iranian elderly nursing homes residents. 
lence of older adults who are at risk of malnutrition among institutionalized elderly in Iran may even be higher than the mentioned report, i.e., the prevalence of malnutrition among elderly residents in nursing home of the Kahrizak Charity Foundation (KCF) was expressed to be as high as $41.3 \%$ (35.0\% among elderly men and $50.3 \%$ in old women) (5).

We need to apply some reliable and valid screening tools for assessing the nutritional status among elderly residents in the long-term care settings (6). These instruments should be easy to use for nursing staffs (7) that should not take longer than 5-15 minutes (6). Furthermore, these screening tools should assess the risk factors of under-nutrition among the elderly group (7).

The Persian version of Mini Nutritional Assessment (MNA) usually is utilized for malnutrition screening in Iranian older adults similar to other countries $(8,9)$. This tool has several items that some of them need anthropometric measurements such as height, weight. In older people particularly those with the inability to move, anthropometric measurements are very difficult (1). A newly introduced instrument for nutritional screening in the elderly is the Nutritional Form for the Elderly (NUFFE). The original version of the NUFFE was developed in Sweden. The NUFFE is a simple instrument that could easily be applied by health providers without special nutritional training and experience. This questionnaire estimates of nutritional status by asking from elderly or their caregivers and application of NUFFE does not need to any measurement (10). The instrument can also be used for both institutionalized (6) and community-dwellers older adults $(10,11)$.

This study aimed to carry out a psychometric evaluation of the Persian version of NUFFE (NUFFE-P) among elderly residents in nursing homes. We hypothesized that the NUFFE-P has sufficient psychometric properties in order to be used as a nutritional screening instrument among the older Iranian population.

\section{Methods}

\section{Study design and sample}

Ninety-seven elderly residents of the Kahrizak Charity Foundation's (KCF) nursing home were enrolled in this cross-sectional study. The KCF is located in the Southern suburbs of Tehran that is the largest nursing home in Iran with more than 1000 older adult residents from a diverse ethnicity. The participants were selected randomly based on a stratified cluster-sampling framework. The wards were the clusters (there were 11 wards in KCF that older adults were living there and the sample was selected from all of these wards weight to number of residents) and the residents were stratified based on the nutritional status using the Mini Nutritional Assessment (MNA) tool that categorized to well-nourished (about $35 \%$ of the participants), at risk of under-nutrition (about 50\% 0f the participants), and under-nourished (about $15 \%$ of the participants). Subjects with MNA score $\geq 24$ considered as well nourished, subjects with scores 17-23.5 considered as at risk of under-nutrition and those with MNA score $<17$ was considered as under-nourished. (8) The expert nurses selected the elderly residents, who met the inclusion criteria. The inclusion criteria were age $\geq 60$ years old, being a resident of the KCF nursing home during the last 6 months and having the ability to communicate in the Persian language. Exclusion criteria included cognitive impairment [Mini Mental State Examination (MMSE) score $\leq 22$ ] (12), having depression [Geriatric Depression Scale-15 (GDS-15) $\geq 8$ ] (13), severe hearing loss that could not communicate in interview (whisper test $<12 / 24$ ) (14), having problems in upper and lower extremities such as a plastering, or amputation that made limitations in anthropometric measuring also to control the effect of acute disease on nutritional status, having a history of hospital admission during 6 months before enrollment.

The sample size was estimated 96 based on the power of $90 \%, \alpha=0.05$ and the Spearman's correlation coefficient of 0.5 between the NUFFE and MNA scores. The subjects assigned to each group according to the distribution weight of nutritional condition in KCF (48 subjects were well nourished, 34 subjects were at risk of under-nutrition, and 14 subjects were undernourished).

All steps of this study were conducted according to the Declaration of Helsinki. The research ethics committee of the Aging Research Center at the University of Welfare and Rehabilitation Sciences (UWRS) in Tehran approved this study (EC-00112). All participants signed the consent form at the beginning of the study and after the orientation session.

\section{Translation process}

After receiving permission from the developer (6), the English version of the NUFFE was translated into the Persian language based on the modified protocol of the International Quality of Life Questionnaire (IQOLA) (15). Two translators, who were professional in English language and were native in Persian, translated the English version of the NUFFE into the Persian language separately. They listed some possible conceptual translations for each item and offered choices. Then they reached an agreement on the translated items and the choices. After that, 10 educated elderly, as well as, 10 formal caregivers assessed the difficulty of the items, using a 100-mm Linear Analog Scale Assessment (LASA) which 100 indicated "extremely difficult" and zero indicated "not at all difficult". The mean difficulty scores $\leq 25$ was considered acceptable (15). Two other translators rated the quality of the translation (0-100) in terms of clarity, the common language applied, and the conceptual equivalence. The quality scores $\geq 90$ was considered an acceptable quality for every three domains (15). Two further translators performed a backward translation. Another English specialist compared the final backward translated instrument with the English version of the NUFFE.

\section{Data collection}

Trained nurses and a nutritionist interviewed the elderly participants in the nursing home. They used a questionnaire to gather demographic data such as age and sex, which was approved by two expert researchers.

Anthropometric measurements: Weight was measured 
in the morning before the breakfast on a leverage scale to the nearest $0.1 \mathrm{~kg}$. Height was measured using a special liner scale ruler that was fixed into the wall in the standing position without any footwear. If the participants were not able to stand up, the measurements were conducted using equipment which could fix the head and the feet in the supine position. The height was measured to the nearest $0.5 \mathrm{~cm}$. Body Mass Index (BMI) was calculated (weight in kilograms divided by height in meters squared). A flexible tape measure was applied to measure the circumferences. Mid Arm Circumference (MAC) was measured at the midpoint of the arm between the tips of the acromion and the olecranon position. The maximum circumference around the calf was considered as a Calf Circumference (CC). These measurements were carried out on the nondominant arm and the lower limb, unless the arm or leg was paralyzed.

Other measurements: A trained researcher evaluated some aspects of the mental and physical health status by administering the MMSE, GDS-15, and Barthel Index. MMSE is one of the most commonly used instruments to assess cognitive status among old people (16). It consists of four domains and 18 items. The maximum and minimum scales of the MMSE are 30 and zero, respectively. Higher scales indicate a better cognitive function (16). Seyedian et al. carried out the psychometric evaluation of the Persian version of the MMSE instrument (12). They reported a cut-point $\leq 22$ to screen the cognitive impairment among the Iranian elderly.

GDS-15 is an instrument to screen of depression among the old population (13). The maximum scale of GDS-15 is 15 , which indicates the worst mood status. This tool was utilized in Iranian older population that was reported a cut-point $\geq 8$ to screen depression mood (13). The Barthel Index is an ordinal scale, which consists of 10 items that measure daily function ability, particularly in daily activities. The total score of the modified form of the Barthel Index is 100 , in which a higher score indicates higher functional independence in activities of daily living (17).

Laboratory tests: Laboratory finding such as serum level of cholesterol, albumin, total protein, and hemoglobin were measured as indicators of nutritional status in older adults $(18,19)$. Venous blood samples as were collected by using a blood collection needle holder after at least 12 hours overnight fasting. The serum was separated from the clot by centrifugation at 6000 RPM, meanwhile, the serum specimens were stored at $-70^{\circ} \mathrm{C}$. Cholesterol and albumin were measured by an enzymatic colorimetric technique with an auto-analyzer (HITACHI R-V910, Japan, and Pars Azmun kits). Hemoglobin levels were measured via HYCEL 300 (France) diagnosis cell counter in whole Ethylenediaminetetraacetic acid (EDTA) blood sample.

Dietary Assessment: In order to assess the association between NUFFE score and total calorie also total protein intake, dietary intake was assessed within 3 days (20). The mean of three days' kilocalorie and protein intake were considered as criteria for nutritional status. These three days included two working days and one holiday. The dietary record describes a 24-hour intake report from midnight to midnight. This dietary record was validated in the
Iranian Household Food Consumption survey (21). A trained dietitian recorded the amount of food intake from the participants. The household dish meal sizes were converted to grams. All served food and beverages, then, were coded according to the prescribed protocol and were analyzed to the content of energy and the other nutrients by Nutritionist III software (version 7.0; N-Squared Computing, Salem, OR), which was designed for Iranian foods (22).

Nutritional assessment instruments: The NUFFE has 15 three-point ordinal scale items. The maximum total score is 30 for the worst, and the minimum total score is zero for the best nutritional condition, i.e. higher scores indicate more risk of under-nutrition. It contains three domains: dietary history (two questions; weight loss and changes in dietary intake), dietary assessment (nine questions; intake of prepared food and fluid, and difficulties to eat), and general assessment (four questions; the possibility of obtaining food products, company to meals, activity and number of medications).

The MNA is composed of 18 items relating to anthropometric measurements (BMI, MAC, and CC), questions about appetite, weight loss, mobility, psychological stress or acute diseases, neuropsychological diseases, type of dwelling, medication, pressure sores or skin ulcers, number of meals, food and fluid intakes, autonomy of feeding and self-perception of health and nutrition. The maximum score is 30 . Scoring categorized the subjects as being well-nourished (24-30 points), at risk for under-nutrition (17- 23.5 points), or undernourished ( $<17$ points). The MNA can categorize the nutrition status of the elderly well without using biochemical indicators (7). Nestlé Company translated the Persian version of the MNA (23). Some studies reported that the Persian version of this tool had relatively good validity and reliability $(5,24)$.

\section{Validity and reliability assessment}

For assess of face validity, three nutritionists scored each item of the NUFFE-P in terms of relevancy of each item to the domains of the nutritional status and clarifying the items in the 3 -point Likert scale $(1=$ poor, $2=$ intermediate, and $3=$ high). The total score of the relevancy and clarifying was equal to 45 . The mean of these two scores was reported as face validity.

Criterion validity was assessed by estimating correlation coefficients between the total NUFFE-IPR score and anthropometric indices such as BMI, CC, MAC, Barthels index, serum albumin, cholesterol, hemoglobin, GDS-15, and a three-day mean of the total calorie and protein intake. A correlation coefficient $\geq 0.7$ was considered as strong, 0.4-0.6 as acceptable, and lower than 0.3 measured as a weak correlation (25).

Concurrent validity was considered as the correlation coefficient between the total NUFFE-P score and the total MNA score. To measure the construct validity, an exploratory factor analysis with Varimax rotation was conducted (26). The median scores of NUFFE-P were compared between "known groups" with expected high and low scores such as those who had BMI $<24 \mathrm{~kg} / \mathrm{m}^{2}$ vs. those who had $\mathrm{BMI} \geq 24 \mathrm{~kg} / \mathrm{m}^{2}$ (27), who had $\mathrm{CC}<31 \mathrm{~cm}$ vs. $\mathrm{CC} \geq 31$ 
$\mathrm{cm}(28)$, and who had $\mathrm{MAC} \leq 23.5$ vs. who had MAC $>$ 23.5 (29).

The internal consistency of this tool was assessed by the item-total correlation and using Cronbach's alpha coefficient. The Cronbach's alpha coefficient $\geq 0.9$ was considered as excellent, $0.7 \leq \alpha<0.9$ as good, $0.6 \leq \alpha<0.7$ as acceptable, $0.5 \leq \alpha<0.6$ as poor, and $\alpha<0.5$ considered as an unacceptable internal consistency (30). The interraters' reliability was assessed by repeating of administration of the Persian version of NUFFE by two nutritionists over a week. The intraclass correlation coefficient (ICC) type $(2, \mathrm{k})$ between the scores assigned to each item and the total NUFFE-P score by two raters among the same participants (31).

Sensitivity, specificity, and positive and negative predictive values were estimated for NUFFE-P with MNA as a criterion. Receiver Operating Characteristic (ROC) curves were constructed with MNA as a criterion in order to find suitable cut-off points to NUFFE-P for identifying individuals at medium or high risk of under-nutrition. The accuracy rate was calculated for a proportion of the participants, who were correctly nutritionally classified by the NUFFE-P based on the MNA, categorized by the total participants (multiplied by 100). Moreover, the BMI $<24$ $\mathrm{kg} / \mathrm{m}^{2}$ and Albumin $<4 \mathrm{~g} / \mathrm{dl}$ considered as criterions to detect of at high risk of under-nutrition by ROC curve analysis.

\section{Statistical analyses}

All analyses were considered statistically significant at $\alpha<0.05$ level and performed by using SPSS 21 (PASW statistics data editor, Chicago). Normality of the distribution of data was assessed using the Kolmogorov-Smirnov Test. For variables without a normal distribution, Spearman's correlation coefficient was used to rely on the strength of relationships. Exploratory factor analysis was utilized to determine factors that construct of the Persian NUFFE tool. The ROC curve analysis was applied to detect the cutoff of NUFFE score based on different criteria. Internal consistency was indicated the Cronbach's alpha coefficient. Inter-rater agreement was assessed using the intraclass correlation coefficient.

\section{Results}

Ninety-seven elderly residents (49 women and 48 old men) of the KCF participated in this study. The range of the ages was from 60 to 100 years. The total NUFFE-P score ranged between 2 and 14 and its mean value was equal to 7.08 [standard deviation (SD): 3.97]. On average, participants had a calorie intakes equal to 1864.70 $(\mathrm{SD}=377.15) \mathrm{Kcals} /$ day and consumptions of 56.07 $(\mathrm{SD}=10.05)$ grams/day protein. General characteristics of the participants are presented in Table 1.

Table 1. General characteristics of the participants $(\mathrm{N}=97)$

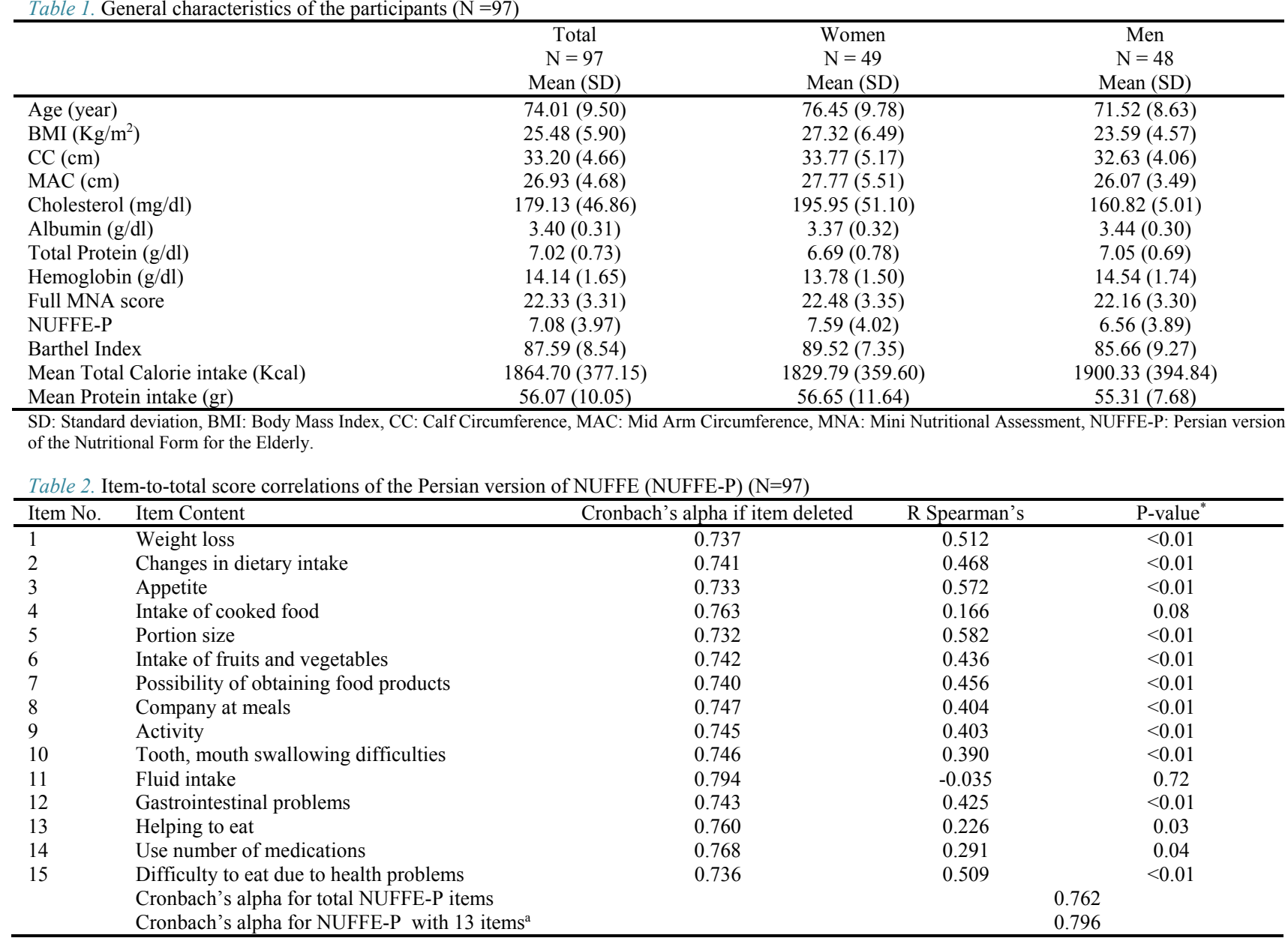

${ }^{\mathrm{a}}$ Intake of cooked food $\&$ fluid intake were deleted. ${ }^{*} \mathrm{P}$ values $<0.05$ were considered statistically significant. 


\section{Face validity}

The mean score of relevancy that was assigned by the three nutritionists was 39.67 of $45(88.15 \%)$, and the mean score of clarifying was 41.67 of $45(92.59 \%)$.

\section{Reliability}

Internal consistency of NUFFE-P was revealed by a Cronbach's alpha coefficient of 0.76 . There was a significant item-total correlation between 13 out of 15 items. After deleting the two items, "intake of cooked food" and "fluid intake", the total Cronbach's alpha coefficient increased (Table 2). Inter-rater reliability of NUFFE-P was reflected by ICC coefficient of 0.98 between the total scores in two times administrated of NUFFE-P by the two raters during one week. The ICC coefficients between two times item scores are presented in Table 3.

\section{Criterion-related validity}

Correlations between the total NUFFE-P score and anthropometric characteristics such as BMI, MAC, CC, also Barthel Index as a functional status, total calorie and pro- tein intakes, and special laboratory indices like serum albumin, cholesterol, and hemoglobin are presented in Table 4. Only the total protein intake did not have a significant correlation with the NUFFE-P score. The strongest correlation was seen between the total NUFFE-P scores and the total calorie intake, and CC.

\section{Concurrent validity}

Spearman rank correlation between the total NUFFE-P score and the total MNA score was $-0.75(\mathrm{p}<0.01)$.

\section{Construct validity}

There were significant differences between the NUFFE$P$ median scores as "known groups" with the expected low and high scores (Table 5).

The exploratory factor analyses revealed four factors, nutritional decline, health problem, intake problem, and other problems (Table 6). The first factor could explain $56.8 \%$ of the variance and contained the items: weight loss, amount of food intake, appetite, and portion size. The Cronbach's alpha coefficient of this factor was 0.845 .

Table 3. Agreements between two raters using the Persian version of NUFFE (NUFFE-P) by interclass correlation coefficient (ICC) (2, K) (N=97)

\begin{tabular}{lcc}
\hline Items & ICC & CI of Intraclass correlation coefficient \\
\hline 1 Weight loss & 0.853 & $0.787-0.899$ \\
2 Changes in dietary intake & 0.907 & $0.864-0.937$ \\
3 Appetite & 0.888 & $0.837-0.924$ \\
4 Intake of cooked food & 0.744 & $0.640-0.821$ \\
5 Portion size & 0.951 & $0.927-0.967$ \\
6 Intake of fruit and vegetables & 0.953 & $0.930-0.968$ \\
7 Possibility of obtaining food products & 0.984 & $0.977-0.990$ \\
8 Company at meals & 0.940 & $0.912-0.959$ \\
9 Activity & 0.963 & $0.946-0.975$ \\
10 Tooth, mouth swallowing difficulties & 0.968 & $0.952-0.978$ \\
11 Fluid intake & 0.967 & $0.951-0.978$ \\
12 Gastrointestinal problems & 0.950 & $0.926-0.966$ \\
13 Helping to eat & 0.846 & $0.778-0.894$ \\
14 Number of medications & 0.960 & $0.941-0.973$ \\
15 Difficulty to eat due to health problems & 0.979 & $0.969-0.986$ \\
Total score of NUFFE-P & 0.982 & $0.971-0.987$ \\
\hline
\end{tabular}

NUFFE-P: Persian version of the Nutritional Form For the Elderly, CI: Confidence Interval, ICC: Intraclass Correlation Coefficient

Table 4. Spearman's correlation coefficient between the total scores of the Persian version of NUFFE (NUFFE-P) and some characteristics of the participants $(\mathrm{N}=97)$

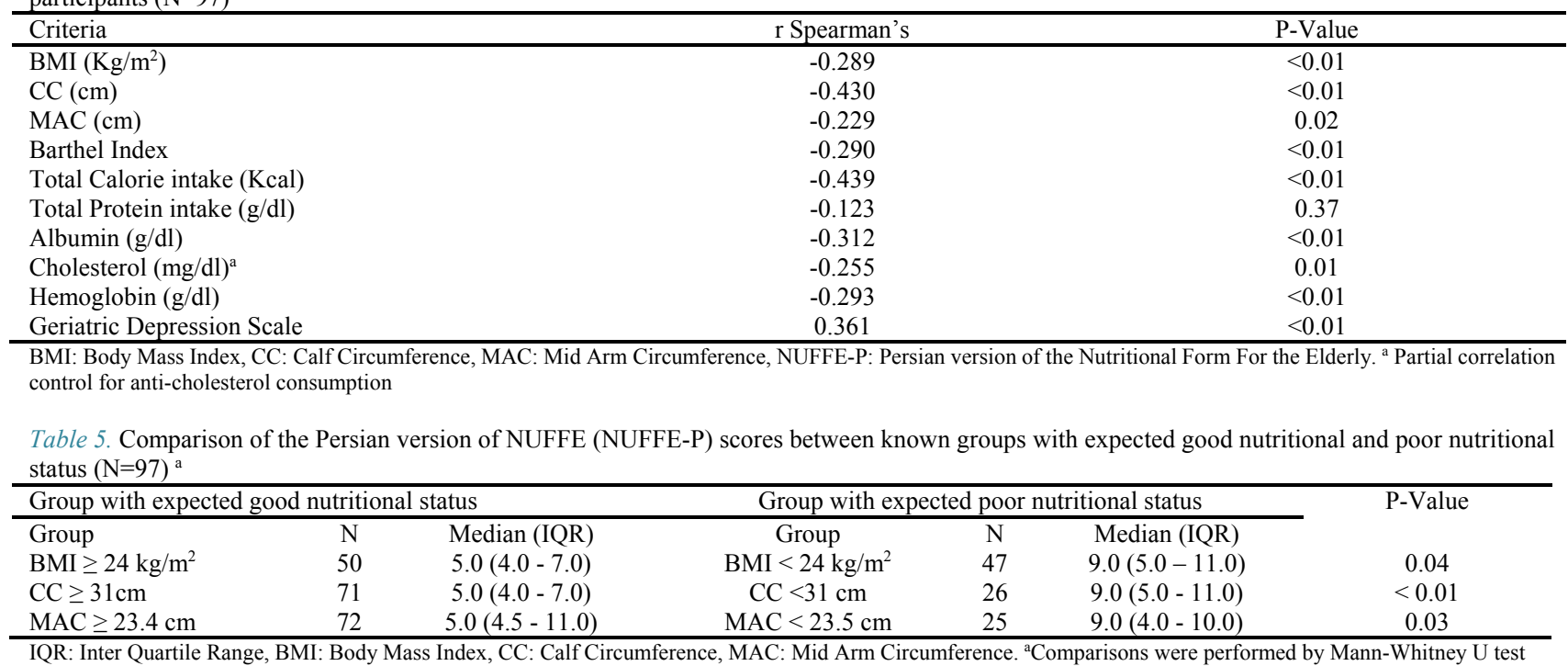


Table 6. The exploratory factor analyses of the Persian version of NUFFE (NUFFE-P)

\begin{tabular}{|c|c|c|c|c|}
\hline & Component 1 & Component 2 & Component 3 & Component 4 \\
\hline & Nutritional Decline & Health Problem & Intake Problem & Other Problems \\
\hline Change in dietary intake & .872 & .036 & -.118 & .161 \\
\hline Appetite & .843 & .129 & .197 & .015 \\
\hline Portion size & .752 & .239 & .241 & -.063 \\
\hline Weight loss & .730 & .256 & -.127 & .138 \\
\hline Exercise & .179 & .692 & -.211 & .165 \\
\hline Intake of fruit and vegetables & .148 & .649 & -.083 & .196 \\
\hline Company at meals & .160 & .589 & -.026 & .130 \\
\hline Health problems & .232 & .574 & .182 & .155 \\
\hline Helping to eat & -.081 & .538 & .161 & -.234 \\
\hline Tooth, mouth swallowing problem & .175 & .504 & .449 & -.396 \\
\hline Gastrointestinal problems & .272 & .441 & .398 & -.199 \\
\hline Fluid intake & -.150 & -.214 & .746 & .101 \\
\hline Access to food products & .312 & .182 & .607 & .297 \\
\hline Intake of cooked food & .076 & .018 & .053 & .709 \\
\hline Number of medications & .087 & .268 & .125 & .659 \\
\hline
\end{tabular}

Extraction Method: Principal Component Analysis.

Rotation Method: Varimax with Kaiser Normalization.

Rotation converged in 22 iterations.

Kaiser-Meyer-Olkin Measure of Sampling Adequacy $=0.760$

Bartlett's Test of Sphericity

Approx. Chi-Square $=392.386, \mathrm{P}<0.0001$

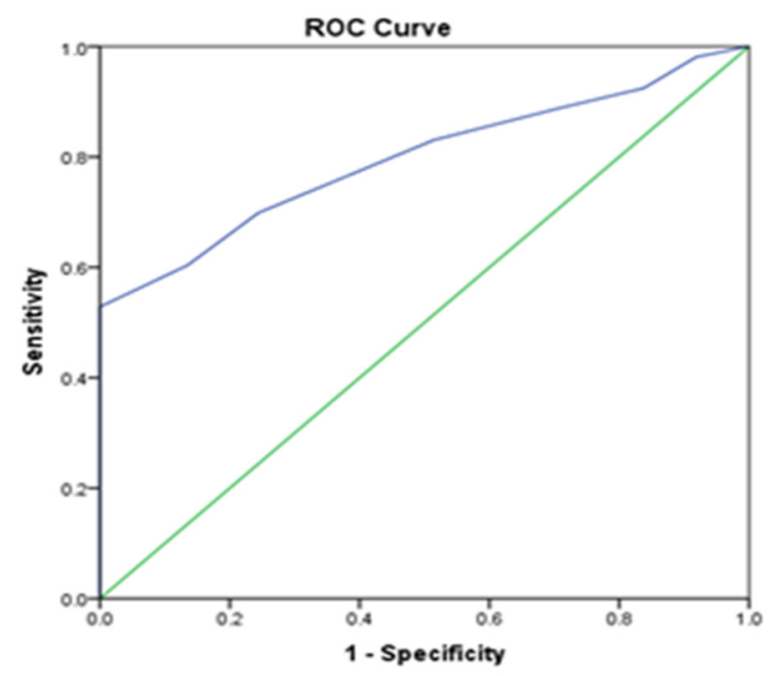

Fig. 1. ROC curve of the Persian version of NUFFE (NUFFE-P) with MNA as criterion for medium risk of under-nutrition at the cut-off point 6 (area under the curve $=0.796$; confidence interval $95 \%=$ $0.705-0.886)$

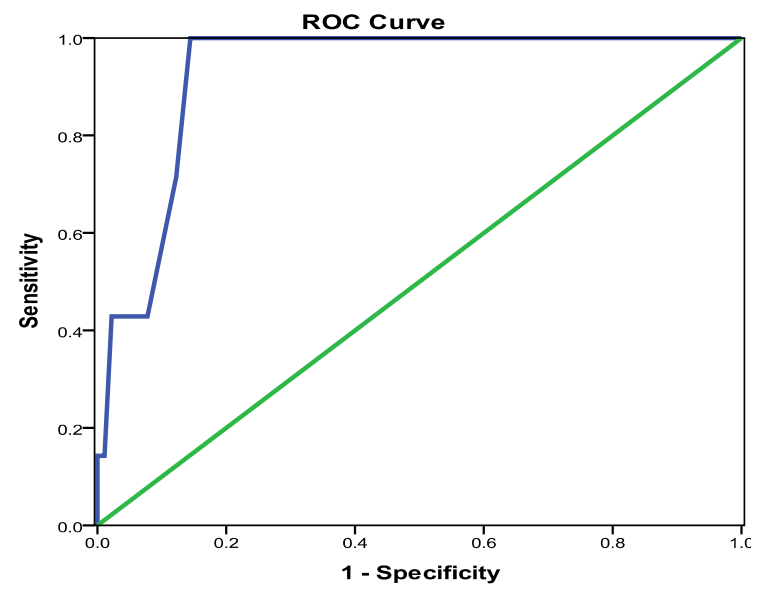

Fig. 2. ROC curve of the Persian version of NUFFE (NUFFE-P) with MNA as criterion for high risk of under-nutrition at the cut-off point 11 (area under the curve $=0.929$; confidence interval $95 \%=0.871-$ $0.986)$

\section{Sensitivity and specificity}

Using MNA as a criterion to NUFFE-P, the area under the ROC curve to detect of medium risk for undernutrition was 0.796 (CI: $0.705-0.886$ ) at the cut-off point of 6.0 (Fig. 1). The calculated sensitivity and specificity values obtained $69.8 \%$ and $75.7 \%$, respectively for detecting those who were at medium risk of under-nutrition. To distinguish the subjects who were at high risks of undernutrition, sensitivity was $100 \%$, and specificity was $85.6 \%$ at the cut-off point of 11.0 (the area under the ROC curve $=0.929$; CI $0.871-0.986$ ) at (Fig. 2). The positive predictive values were calculated as $80.8 \%$, and $30.3 \%$ and the negative predictive values were estimated as $60.4 \%$ and $71.4 \%$ to detect of individuals at medium risk for undernutrition and at high risk for under-nutrition.

The total accuracy rate was equal to $59.8 \%$. The cut-off points of NUFFE-P, for detection of high risk of undernutrition, with serum albumin level $<4.0 \mathrm{~g} / \mathrm{dl}$ and BMI $<$ $19 \mathrm{~kg} / \mathrm{m}^{2}$ as criterions were 11.0 and 10.0 , respectively (Table 7).

\section{Discussion}

This study was designed to evaluate the psychometric properties of the NUFFE-P. Our findings revealed that the NUFFE-P has sufficient evidence of reliability and validity, including sensitivity and specificity, to identify the elderly who are at a medium risk of under-nutrition and those who are at high risk of under-nutrition in nursing home settings. Thereby the hypothesis was accepted.

Based on the results, there is a good internal consistency of the NUFFE-P (30). Internal consistency in this study

Table 7. Sensitivity and specificity of the Persian version of NUFFE (NUFFE-P) for detection of high risk of under-nutrition with MNA, Albumin, and BMI as criterions

\begin{tabular}{lccc}
\hline & $\begin{array}{c}\text { Cut-off } \\
\text { point }\end{array}$ & $\begin{array}{c}\text { Sensitivity } \\
(\%)\end{array}$ & $\begin{array}{c}\text { Specificity } \\
(\%)\end{array}$ \\
\hline MNA $(<17)$ & 11 & 100 & 85.6 \\
Albumin $(<4 \mathrm{~g} / \mathrm{dl})$ & 11 & 75.0 & 84.9 \\
BMI $\left(<19 \mathrm{~kg} / \mathrm{m}^{2}\right)$ & 10 & 84.6 & 76.2 \\
\hline
\end{tabular}

MNA: Mini Nutrition Assessment, BMI: Body Mass Index 
was approximately equal to the levels that were reported by the Norwegian study in institutionalized elderly (6). Streiner and Norman recommend that the items which do not meet an item-total score correlation of 0.20 should be omitted from the questioner's instrument and the items with low variability be excluded from the analysis (32). The internal consistency is increased with deleting the items with low variability. In this study item 4 (intake of cooked food) and item 11 (fluid intake) had weak itemtotal correlation; however, we did not exclude them since the NUFFE was developed as a comprehensive instrument to reflect the number of causal factors to determine undernutrition (33). In other words, these items conceptually had strong relationships with the nutritional status, and exclusion of them is not reasonable. In the Norwegian study (6), 13 items out of 15 items showed statistically significant correlations between 0.20 and 0.60 . In the Hungarian study, the number of significant items-total correlations was less than our study. The item-total score correlations were not significant in 6 items in the Hungarian study (33). A possible explanation of this discrepancy may be due to the difference of food patterns and nutritional behaviors among the Iranian older population in this study compared to the other studies. The justification of the weak item-total correlation regarding the 'eating cooked meal/day' item in our study is the low variability due to the fact that roughly all participants in our study had at least one cooked meal per day that was served at lunch time or dinner. Another explanation for the differences in the items-total correlation (items 4 and 11) may be for the reason that most of the elderly participants in this study did not have the same concept about the word "intake" for the consumption of food or liquid.

We found a strong inter-rater reliability sue to high agreement between scoring of two raters. The intraclass correlation coefficient type $(2, \mathrm{~K})$ that we used is one of the best methods for assessing the agreement in the ordinal data (32). Except for the fourth item (eating cooked meal/day), we found an excellent agreement between the two raters. Gao et al. have recently reported an $\mathrm{ICC}=0.88$ for the Chinese version of NUFFE (10).

Anthropometric procedures are mutual factors for evaluating nutritional status (29), and three anthropometric criteria (BMI, CC, MAC) were used to assess the validity of the NUFFE-P, meanwhile Barthel Index score, total calorie intake, total protein intake, serum albumin, and hemoglobin levels were considered as the other criteria to evaluate the criterion-related validity of the NUFFE-P. The validity was supported throughout significant correlations that were found between the total NUFFE-P scores and all those criteria, except for the total protein intake. However, we could not find any significant association between the NUFFE-P score and the total protein intake. Also a similar lack of the correlation was found between the total protein intake and the total MNA score in our study (data have not been shown). These lacks of correlations may be due to the problem in converting Iranian dish meal sizes to a quantitative measure of the nutrients.

We used the exploratory factor analysis for assessing that the Persian NUFFE is valid in terms of construction.
Several studies have utilized this method for evaluation of construct validity $(26,34)$. We found four components (nutritional decline, health problem, intake problem, and other problems) in the factor analysis of NUFFE-P. These components were different compared to the findings of Gombos et al., which reported six factors for the Hungarian version of NUFFE. However, the first factor was similar in our study and the other study (33). The first factor is the most important factor that could explain more than $56 \%$ of the variation. The Cronbach's alpha coefficient of the first factor was higher than the finding of the Hungarian's study (33).

Concurrent validity was confirmed with a high Spearman's rank correlation coefficient between the NUFFE-P and the MNA total scores. The same high negative correlation between the MNA and NUFFE scores was reported in the Norwegian study (6). The MNA is a well-known tool to assess nutritional status as one of the components of the comprehensive geriatric assessment. Many researchers consider the MNA as the golden standard for nutritional screening in the older adults (7). Since its high concurrent validity, NUFFE-P can be used as a good alternative for the MNA. Applying the NUFFE-P by health providers is also easier than MNA, because it does not require anthropometric measurements. Performing these measurements is usually difficult in aged groups (1).

This study had some limitations. The first was that sampling conducted in a long-term care institute $(\mathrm{KCF})$ and the results had to be cautiously generalized to the general older population. Another limitation might be the small sample size of the study. Performing a simultaneous exploratory and confirmatory factor analysis needs a large data for dividing the participants into two groups, randomly, one group to explore the factors and another group for testing the reliability of the found factors by a confirmatory factor analysis (35). We found an area under the ROC curve near 0.8 regarding the medium risk of under-nutrition and higher than 0.9 concerning the high risk of under-nutrition based on the MNA categorized. Our findings, in comparison to the Norwegian study, are similar in the diagnosis of the medium risk of malnutrition but we observed a higher area under the ROC curve in terms of the diagnosis of malnutrition (6). This higher sensitivity and specificity of the NUFFE-P than the Norwegian version of NUFFE in distinguishing malnutrition may refer to the small number of the malnourished participants in our study or to different sampling frameworks of these studies. The NUFFE-P had a good sensitivity and specificity to detect of high-risk under-nutrition by the criterions serum albumin and BMI.

\section{Conclusion}

The NUFFE-P showed sufficient psychometric properties in research on the older residents in a nursing home. This instrument showed a good homogeneity and interrater reliability as well as a good validity. The high sensitivity and specificity values of this tool revealed that NUFFE-P can particularly detect the older people at high risk of under-nutrition. 


\section{Acknowledgment}

We would like to thank Mr. Mohamad-Reza Soufinejad; deputy of the Kahrizak Charity Foundation institute in his cooperation to carrying out this project.

This study was supported by the University of Social Welfare and Rehabilitation Sciences (No. 108/4/58/62671) and the Kahrizak Charity Foundation (No. A241/1386).

\section{Conflict of Interests}

The authors declare that they have no competing interests.

\section{References}

1. Ahmed T, Haboubi N. Assessment and management of nutrition in older people and its importance to health. Clin Interv Ag. 2010;5:20716.

2. Stange I, Poeschl K, Stehle P, Sieber CC, Volkert D. Screening for malnutrition in nursing home residents: Comparison of different risk markers and their association to functional impairment. J Nutr Health Ag. 2013;17(4):357-63

3. Wijnhoven HAH, Schilp J, de Vet HCW, Kruizenga HM, Deeg DJH, Ferrucci L, et al. Development and validation of criteria for determining undernutrition in community-dwelling older men and women: The short nutritional assessment questionnaire $65^{+}$. Clin Nutr. 2012;31(3):351-8.

4. Ferreira LS, Nascimento LFC, Marucci MFN. Use of the mini nutritional assessment tool in elderly people from long-term institutions of southeast of Brazil. J Nutr Health Ag. 2008;12(3):2137.

5. Mirarefin M, Sharifi F, Fakhrzadeh H, Nazari N, Ghaderpanahi M, Badamchizade Z, et al. Predicting the value of the Mini Nutritional Assessment (MNA) as an indicator of functional ability in older Iranian adults (Kahrizak elderly study). J Nutr Health Ag. 2011;15(3):175-80

6. Söderhamn U, Flateland S, Jessen L, Söderhamn O. Norwegian version of the nutritional form for the elderly: Sufficient psychometric properties for performing institutional screening of elderly patients. Nutr Res. 2009;29(11):761-7.

7. Söderhamn U. Tools to identify nutritional risk for older people in the home. Br J Community Nurs. 2012;17(Sup11):S26-S9.

8. Machado RSP, Coelho MASC, Veras RP. Validity of the Portuguese version of the mini nutritional assessment in Brazilian elderly. BMC Geriatr. 2015;15:132-9.

9. Fossum M, Terjesen S, Ehrenberg A, Ehnfors M, Söderhamn O. Evaluation of the Norwegian version of the Mini Nutritional Assessment (MNA) among older nursing home patients. Nord J Nurs Res. 2009;29(2):50-2.

10. Gao H, Söderhamn U, Zhang L, Cui H-X, Liu K. Reliability and validity of the Chinese version of the Nutritional Form For the Elderly. Public Health Nutr 2015;18(14):2559-64

11. Söderhamn U, Dale B, Sundsli K, Tomstad ST, Söderhamn O. Psychometric testing of the Norwegian version of the nutritional form for the elderly among older home-dwelling people. J Multidiscip Health. 2012;5:121-8.

12. Seyedian M, Falah M, Nourouzian M, Nejat S, Delavar A, Ghasemzadeh HA. Validity of the Farsi version of mini mental state examination. J Med Counc Iran. 2008;25(4):408-14. [Persian].

13. Khesali Z, Mohammadi Shahboulaghi F, Khanke H, Biglarian A. Effect of "Tai Chi Chuan" on Depression in elderly women. Iran J Rehabil Res Nurs. 2015;2(2):39-46. [Persian]

14. Gibson WK, Cronin H, Kenny RA, Setti A. Validation of the selfreported hearing questions in the Irish longitudinal study on ageing against the Whispered Voice Test. BMC Res Notes. 2014;7:361-7.

15. Squires A, Aiken LH, van den Heede K, Sermeus W, Bruyneel L, Lindqvist $\mathrm{R}$, et al. A systematic survey instrument translation process for multi-country, comparative health workforce studies. Int J Nurs Stud. 2013;50(2):264-73.

16. Ansari NN, Naghdi S, Hasson S, Valizadeh L, Jalaie S. Validation of a Mini-Mental State Examination (MMSE) for the Persian population: A pilot study. Appl Neuropsychol. 2010;17(3):190-5.
17. Quinn TJ, Langhorne P, Stott DJ. Barthel index for stroke trials: Development, properties, and application. Stroke. 2011;42(4):114651.

18. Zhang Z, Pereira SL, Luo M, Matheson EM. Evaluation of blood biomarkers associated with risk of malnutrition in older adults: A systematic review and meta-analysis. Nutrients. 2017;9(8):829-48.

19. Cabrerizo S, Cuadras D, Gomez-Busto F, Artaza-Artabe I, MarínCiancas F, Malafarina V. Serum albumin and health in older people: Review and meta analysis. Maturitas. 2015;81(1):17-27.

20. Ortega RM, Pérez-Rodrigo C, López-Sobaler AM. Dietary assessment methods: Dietary records. Nutr Hosp. 2015;31(Supl.3):3845.

21. Abdollahi M, Mohammadi-Nasrabadi F, Houshiarrad A, Ghaffarpur M, Ghodsi D, Kalantari N. Socio-economic differences in dietary intakes: The comprehensive study on household food consumption patterns and nutritional status of I.R. Iran. Nutr Food Sci Res. 2014;1(1):19-26

22. Azadbakht L, Esmaillzadeh A. Macro and micro nutrients intake, food groups consumption and dietary habits among female students in Isfahan university of medical sciences. Iran Red Crescent Med J. 2012;2012(4, Apr):204-9.

23. Nestle Nutrition Institute. Persian version of mini nutritional assessment. Nestle Nutrition Institute; [updated 2018.02.25; cited 2018.02.25].Available from: http://www.mna-elderly.com/note_farsi sf.html.

24. Amirkalali B, Sharifi F, Fakhrzadeh H, Mirarefin M, Ghaderpanahi M, Larijani B. Evaluation of the mini nutritional assessment in the elderly, Tehran, Iran. Public Health Nutr. 2010;13(09):1373-9.

25. Mukaka MM. Statistics Corner: A guide to appropriate use of correlation coefficient in medical research. Malawi Med J. 2012;24(3):69-71

26. Besnoy KD, Dantzler J, Besnoy LR, Byrne C. Using exploratory and confirmatory factor analysis to measure construct validity of the Traits, Aptitudes, and Behaviors Scale (TABS). J Educ Gift. 2016;39(1):3-22

27. Babiarczyk B, Turbiarz A. Body Mass Index in elderly people-Do the reference ranges matter? Prog Health Sci. 2012;2(1):58-67.

28. Cuervo M, Ansorena D, García A, González MMA, Astiasarán I, Martínez JA. Assessment of calf circumference as an indicator of the risk for hyponutrition in the elderly. Nutr Hosp. 2008;24(1):63-7. [Spanish]

29. Tang AM, Dong K, Deitchler M, Chung M, Maalouf-Manasseh Z, Tumilowicz A, et al. Use of cutoffs for mid-upper arm circumference (MUAC) as an indicator or predictor of nutritional and health-related outcomes in adolescents and adults: A systematic review. Washington, US: Food and Nutrition Technical Assistance III Project (FANTA); 2013

30. Uysal M, Sirakaya-Turk E. Factor analytical procedure and scale reliability. In: Sirakaya-Turk E, Uysal M, Hammitt WE, Vaske JJ, editors. Research Methods for Leisure, Recreation and Tourism. 2nd ed. USA: CABI; 2017. p. 320-45.

31. Koo TK, Li MY. A guideline of selecting and reporting intraclass correlation coefficients for reliability research. J Chiropr Med. 2016;15(2):155-63.

32. Streiner DL, Norman GR. Health measurement scales: A practical guide to their development and use. 4th ed. New York, US: Oxford University Press; 2008. $450 \mathrm{p}$.

33. Gombos T, Kertész K, Csíkos Á, Söderhamn U, Söderhamn O, Prohászka Z. Nutritional form for the elderly is a reliable and valid instrument for the determination of undernutrition risk, and it is associated with health-related quality of life. Nutr Res. 2008;28(2):59-65

34. Atkinson TM, Rosenfeld BD, Sit L, Mendoza TR, Fruscione M, Lavene D, et al. Using confirmatory factor analysis to evaluate construct validity of the Brief Pain Inventory (BPI). J Pain Symptom Manage 2011;41(3):558-65

35. Myers ND, Ahn S, Jin Y. Sample size and power estimates for a confirmatory factor analytic model in exercise and sport: A Monte Carlo approach. Res Q Exerc Sport 2011;82(3):412-23 